\title{
Experimental Mathematics
}

\section{Searching for Hyperbolic Polynomials with Span Less than 4}

\section{Stefano Capparelli, Alberto Del Fra \& Andrea Vietri}

To cite this article: Stefano Capparelli, Alberto Del Fra \& Andrea Vietri (2020): Searching for Hyperbolic Polynomials with Span Less than 4, Experimental Mathematics, DOI: 10.1080/10586458.2019.1706670

To link to this article: https://doi.org/10.1080/10586458.2019.1706670

册Published online: 11 Jan 2020.

Submit your article to this journal $₫$

山 Article views: 20

Q View related articles $\widetilde{ }$

View Crossmark data $\nearrow$ 


\title{
Searching for Hyperbolic Polynomials with Span Less than 4
}

\author{
Stefano Capparelli, Alberto Del Fra, and Andrea Vietri \\ Department of Basic and Applied Sciences for Engineering, Sapienza University of Rome, Rome, Italy
}

\begin{abstract}
A monic, irreducible polynomial in one variable having integer coefficients and all real roots deserves particular interest if its roots lie in an interval of length 4 whose end-points are not integers. This follows by some pioneering studies by R. Robinson. Thanks to the crucial support of computers, a number of contributions over the decades settled the existence question for such polynomials up to degree 18 . In this article, we find out that almost all of these polynomials can be recovered with algebraic operations from a few polynomials of small degree. Furthermore, a great number of the polynomials discovered by Robinson can be actually obtained as simple linear combinations of Chebyshev polynomials. As a byproduct, we found several families of hyperbolic polynomials related to Salem's numbers.
\end{abstract}

\section{KEYWORDS}

Algebraic integer;

hyperbolic polynomial;

Chebyshev polynomial

MSC (2010)

11C08; 11Y99; 12D10

\section{Introduction}

The present article follows the research line that started with the pioneering work [8] by Robinson and was continued by several other authors-see $[1,2,3,6]$; our main object of study are therefore monic, irreducible polynomials in one variable having integer coefficients and all real roots (i.e., hyperbolic), whose span (the smallest interval containing the roots) is required to be smaller than 4 , excluding the case where the roots lie in an interval of length 4 with integer end-points. The importance of the threshold 4 for the span will be recalled in the next lines. Let us denote by $\mathcal{N}$ the set of all known polynomials with the mentioned properties (see Appendix A). Naturally, these polynomials are considered up to substitutions of the form $x \rightarrow-x$ and $x \rightarrow x+a$ for any integer $a$ (see $[1,3,8])$. The novelty in our approach is that the construction of such polynomials avails of purely algebraic operations (linear combinations and products of suitable polynomials, with the unity 1 included) which can be performed inside the whole algebra of polynomials in one variable. As an emblematic example, let us consider one of the polynomials in $\mathcal{N}$ with degree 18 (the largest known degree) that were remarkably found in [2] using integer linear programing-see $f_{156}$ in Appendix A. In their article, the authors assumed some inequality constraints on the coefficients so as to reduce the computational complexity; in particular, they reasoned on the typical distribution of the roots of the already known polynomials. As the authors themselves noticed, when using linear programing in this context it is inevitable that the complexity dramatically increases as the degree increases; this is the reason why polynomials of higher degrees-should they exist-are still hard to find. However, using the present approach it turns out that $f_{156}$ is simply equal to $f_{2} f_{8} f_{15} f_{31}-1$; there is also another way of obtaining $f_{156}$, namely as $f_{31} f_{32} f_{33}-f_{31}-$ 1. This is an example of what happens in several cases, namely, that many high degree polynomials in $\mathcal{N}$ can be obtained by very simple algebraic manipulations of small degree polynomials in $\mathcal{N}$. As a further example we have that $f_{150}$, whose degree is 16 , occurs as a factor of $f_{136}^{2}+$ $f_{136}-1$, where $f_{136}$ has degree 14 . Although in this case we do not obtain exactly $f_{150}$, factoring a polynomial is an elementary operation from the computational viewpoint, provided that the degree is not too high. As this example shows, a polynomial expression involving a single polynomial can succeed in yielding a new example, possibly by the use of factorization.

With the above examples in mind it seems reasonable to search for an optimal subset of polynomials in $\mathcal{N}$ which may generate other polynomials in $\mathcal{N}$ itself by means of straightforward combinations, using suitable sums and products. In this spirit, the request that the generating family lie inside $\mathcal{N}$ itself could be relaxed to obtain a more general approach. Indeed, in Sections 3 and 4 we do not confine ourselves to $\mathcal{N}$ and consider the external family of Chebyshev polynomials $\left\{c_{0}, c_{1}, \ldots\right\}$ as a basis for the space $\mathbb{R}[x]$. By doing so, for example, it turns out that $f_{40}=$ $c_{0}-c_{1}-c_{5}+c_{6}$ and $f_{77}=-c_{1}-c_{3}-c_{5}+c_{8}$. Notably, linear combinations with coefficients in $\{-1,0,1\}$ are enough to obtain 20 polynomials out of the 77 that already appeared in [8]. Other regularities were observed such as alternating signs and a weak monotonicity, in absolute value. For example, $x^{8}-3 x^{7}-5 x^{6}+18 x^{5}+7 x^{4}-33 x^{3}-3 x^{2}+18 x+$ 1 has coordinates $[7,-6,6,-6,5,-3,3,-3,1]$, and one 
of the three polynomials of degree 18 found in [2] it is exactly of this form:

$$
\begin{aligned}
& {[15,-15,15,-14,14,-13,12,-11,10,-9,8,} \\
& -7,6,-5,4,-3,2,-2,1] .
\end{aligned}
$$

Let us step back and provide some details which pave the way for the present analysis. According to a classical result of Kronecker's (see [4]), a set of conjugate algebraic integers lying on the unit circle $|z|=1$ consists of roots of unity. After transforming the unit circle, $|z|=1$, into the segment $-2 \leq x \leq 2$ via the function $x=z+\frac{1}{z}$, Kronecker obtains that any algebraic integer which lies with its conjugates in the interval $[-2,2]$ must be of the form $x=2 \cos \frac{2 k \pi}{m}$. Following the literature, the corresponding polynomials, which are all irreducible, will be called polynomials of cosine type or also Kronecker polynomials. In this article, we call polynomials having all roots in the interval $[-2,2]$, but not necessarily irreducible, polynomials of Kronecker type. Now we come to the mentioned threshold 4 for the span. In [7] (see also [9]) R. Robinson writes that G. Pólya and I. Schur (see [13]) showed that a real interval of length less than 4 can contain only a finite number of sets of conjugate algebraic integers, and then proceeds to prove that any real interval of length greater than 4 contains an infinite number of sets of conjugate algebraic integers. He writes: "The problem remains unsolved for intervals of length exactly 4, except when the end-points are rational integers, in which case there are infinitely many sets". That was the beginning of the research stream on irreducible polynomials with integer coefficients having only real roots, such that the span is less than 4. In [8], Robinson classified all such polynomials for degrees up to and including 8 . He chose the representative polynomials of each type in such a way that the average of the roots lies in $\left[0, \frac{1}{2}\right]$.

Recently, in [1], Robinson's classification was extended up to degree 14, although a list up to degree 17 was obtained and conjectured to be complete. In [3] it was proved that the list is exhaustive up to degree 15. Finally, in [2], with the aid of linear programing the authors seemed to suggest that the list of polynomials of degree 16 and 17 found in previous papers is indeed complete, though no proof of this is yet available. Moreover, as we remarked, the authors exhibited three polynomials of degree 18; in spite of similar computations being conducted, no such polynomials of degree 19 or 20 were found that were not of cosine type. A related research is the one carried out by McKee in [6] which uses integer symmetric matrices.

The present article is organized as follows: In Section 2, we explore algebra operations and search for an optimal set of generators for $\mathcal{N}$, in a sense that we will make precise. In Section 3, we describe an infinite family of Kronecker polynomials. In Section 4, we give some families of polynomials that are related to Salem's and Pisot's numbers.

\section{Working with the algebra of polynomials: The placenta map}

Let us denote by $\mathcal{K}$ the set of Kronecker polynomials. In the present section we introduce a strategy for obtaining some polynomials in $\mathcal{N}$ using polynomials in $\mathcal{N}$ of lower degree, possibly with the contribution of $\mathcal{K}$, and operations in the algebra of polynomials. Although we did not succeed in obtaining new polynomials to add to $\mathcal{N}$, our approach provides a new insight into $\mathcal{N}$. As a starting point we observe that the characterizing properties of polynomials in $\mathcal{N} \cup \mathcal{K}$ might happen to be preserved after performing suitable sums and/or products of some of them. This is justified by a continuity argument which ensures that every resulting polynomial has its roots "very close" (although a priori in $\mathbb{C}$ ) to the roots of the generating polynomials; therefore, it is reasonable to expect that some polynomial $\Phi$ we obtain in this fashion has a factor in $\mathcal{N}$. Notice that other factors need not have real roots and that $\Phi$ need not be monic.

The idea of combining two or more polynomials and subsequently analyzing the roots of the outcome is not at all recent; authors such as Marden (see [5]) and earlier Walsh (see [15]) found notable results albeit for the complex case; transferring their findings to the extremely demanding context of real numbers, with the further restrictions we have on the span, could be a future challenging project.

The list of $\mathcal{N}$ in Appendix $\mathrm{A}$, is ordered by $\prec$ as follows: $f \prec g$ if $f$ has lower degree than $g$, or, if $f$ and $g$ have the same degree, then $f \prec g$ according to the lexicographic order of their coefficients starting form the leading coefficient. We will regard $\mathcal{N}$ as a part of a larger set, $(\mathcal{M}, \prec)$, of representatives of non-Kronecker irreducible hyperbolic polynomials whose span is less than 4 of any degreealthough at present $\mathcal{M} \backslash \mathcal{N}$ is empty!

After choosing a polynomial $\Phi \in \mathbb{Z}\left[x_{1}, \ldots, x_{t}, x\right]$ in $t+1$ variables, we build a map

$$
\mathcal{P}_{\Phi}:(\mathcal{N} \cup \mathcal{K})^{t} \rightarrow(\mathcal{M} \cup\{0\})^{\mathbb{N}}
$$

by associating $\left(g_{1}, g_{2}, \ldots, g_{t}\right)$ to $\left(h_{1}, h_{2}, \ldots, h_{u}, 0,0,0, \ldots\right)$, where the $h_{i}$ 's are all the irreducible factors of $\Phi\left(g_{1}, g_{2}, \ldots, g_{t}, x\right)$ which belong to $\mathcal{M}$, with possible repetitions and in nondecreasing order with respect to $\prec$. For example, if $\Phi=$ $x_{1} x_{2} x_{3}-x$ and $\gamma$ is the Kronecker polynomial $x^{5}-x^{4}$ $4 x^{3}+3 x^{2}+3 x-1$, then, in the notation of Appendix A, $\mathcal{P}_{\Phi}\left(f_{1}, f_{71}, \gamma\right)=\left(f_{133}, 0,0,0, \ldots\right)$ because the resulting polynomial is equal to $\left(x^{2}-3\right) f_{133}$ and the first factor does not belong to $\mathcal{M}$ (in passing, notice that it is nonetheless a Kronecker polynomial).

Definition 1. We call $\mathcal{P}_{\Phi}$ the placenta map defined by $\Phi$. Given $f \in \mathcal{M}$, the map $\mathcal{P}_{\Phi}$ is said to generate $\mathrm{f}$ if $\mathrm{f}$ occurs in some sequence $\mathcal{P}_{\Phi}\left(g_{1}, g_{2}, \ldots, g_{t}\right)$.

As mentioned above, the main question amounts to finding placenta maps which are able to generate polynomials with degree necessarily larger than 15 in $\mathcal{M} \backslash \mathcal{N}$. Typically, one chooses a somewhat "fertile" placenta map and replaces 
each variable $x_{q}$ with some polynomial in $\mathcal{N} \cup \mathcal{K}$. In what follows we record some experimental results which were obtained using the software Mathematica as provided by Sapienza Università, InfoSapienza CAMPUS Software. In spite of the many efforts in our search for new polynomials, all the placenta maps under examination generated only known polynomials (although a great number of them) and this gradually led us to the feeling that probably $\mathcal{M}=\mathcal{N}$. On the other hand, the most gratifying aspect of our search was the discovery of certain algebraic relations among polynomials in $\mathcal{N}$ by virtue of suitable placenta maps; indeed, in the list of 158 elements, created and updated over the decades with the growing aid of computers, this procedure has disclosed some valuable information on the algebraic structure of $\mathcal{N}$ : the elements of $\mathcal{N}$ are no longer solitary fruits of a careful and intense search; the present approach unveils some of their interactions as elements of the algebra of polynomials.

Let us show an emblematic example.

Proposition 2. If $\Phi=x_{1} x_{2}-x_{3}+x_{4}-1$, the placenta map $\mathcal{P}_{\Phi}$ generates all polynomials of degree larger than 4 in $\mathcal{N}$ with the exception of $f_{142}, f_{152}, f_{154}, f_{156}, f_{157}$ whose degrees are $14,16,16,17,18,18$, respectively. In all cases, the degree of the generated polynomial is larger than the degrees of the four polynomials that replace the variables $x_{i}$.

The data which prove the proposition have been collected in Appendix B. This result enables us to recursively generate all but 5 elements of $\mathcal{N}$ with just a single placenta map. It appears, therefore, that $\mathcal{N} \cup \mathcal{K}$ has a rich and interesting structure from an algebraic point of view. According to our findings no other placenta map behaves as thoroughly as the one above.

Of the five missing polynomials, two were found using a different map:

Proposition 3. The placenta map of $x_{1} x_{2} x_{3} x_{4}-1$ generates $f_{156 ;}$ more precisely, $f_{156}=f_{2} f_{8} f_{15} f_{31}-1$. Furthermore, the same placenta map generates $f_{142}$ as a factor of $f_{36} f_{71} f_{89} f_{117}-1$.

Unlike the previous placenta map, it turns out that this generates few interesting polynomials; nonetheless it is useful to fill a gap with relatively little computer effort.

There is something more to notice. Requiring that a polynomial be equal to-and not just a factor of - a suitable combination of certain polynomials is a restriction which precludes the production of many polynomials but, on the other hand, besides speeding up the computation, it allows for a more refined algebraic manipulation. Remarkably, in the case of $f_{156}$ the above identity can be coupled with a further experimental identity which we already mentioned, namely, $f_{156}=f_{31} f_{32} f_{33}-f_{31}-1$. Therefore we have that $f_{156}=f_{2} f_{8} f_{15} f_{31}-1=f_{31} f_{32} f_{33}-$ $f_{31}-1$, which implies that $f_{2} f_{8} f_{15}-f_{32} f_{33}+1=0$, so that one of these last polynomials can be seen as a function of the others. In some other cases it is even possible to express one polynomial as a combination of the others without resorting to rational functions. In the second part of this section we will provide some details in this regard.

Coming back to general placenta maps, let us briefly report on some further experiments. Once a polynomial yields an interesting map, it seems reasonable to generalize it as in the case of $\Phi=x_{1} x_{2} x_{3}+\varepsilon_{1} x_{1} x_{2}+\varepsilon_{2} x_{1} x_{3}+\varepsilon_{3} x_{2} x_{3}+$ $\varepsilon_{4} x_{1}+\varepsilon_{5} x_{2}+\varepsilon_{6} x_{3}+\varepsilon_{7} \quad$ with $\varepsilon_{i} \in\{-1,0,1\}$. When the parameters $\varepsilon_{i}$ vary, the corresponding placenta maps generate in particular $f_{156}$ (in the second way we showed) and other polynomials arise such as $f_{150}$, a factor of $f_{29} f_{31} f_{58}-$ $f_{29} f_{31}+f_{29}-f_{31}-f_{58}+1$. The computational complexity here begins to increase due to the presence of many parameters.

Another fecund family of polynomials is $x_{1} x_{2}+$ $x_{1}\left(\varepsilon_{1} x+\eta_{1}\right)+x_{2}\left(\varepsilon_{2} x+\eta_{2}\right)+\varepsilon_{3} x+\eta_{3}$, for small values of the 6 parameters. It generated many polynomials albeit of degree smaller than 16. Instead, after generalizing the first map which generated $f_{156}$, notably we found that $f_{2} f_{8} f_{15} f_{31}-f_{2} f_{31}-1=x f_{153}$. Alterations using polynomials of degree 2 or more were also fruitful. As a last example, we notice that placenta maps with polynomials in only one variable are a special case deserving some further investigations. For example, $\mathcal{P}_{x_{1}^{2}+x_{1}-1}$ generates $f_{150}$ as already shown.

We have now come to the second clue against the existence of further elements in $\mathcal{M}$. In some experiments, the removal of the constraint of being monic produced a high number of polynomials of degree well larger than 18. For example, it was enough to consider $\mathcal{P}_{2 x_{1} x_{2}-1}$; the polynomials we found had in many cases all even coefficients except for the constant term. Repeating the experiment with, say, $\mathcal{P}_{3 x_{1} x_{2}-1}$ yielded similar results. It is then a peculiar behavior for a placenta map, that of stopping at degree 18 and producing only the polynomials already known, once the requirement for the polynomial to be monic is added.

By the many experiments it emerged that polynomials of small degree in $\mathcal{N}$ play a basic role: indeed, using suitable algebraic manipulations of such polynomials we succeeded in rediscovering almost all known polynomials of large degree. Actually, as we hinted above, small degree polynomials become even more interesting if we restrict our attention to placenta maps which have further properties as in the following definition.

Definition 4. Let $\Phi$ be a monic polynomial in $\mathbb{Z}\left[x_{1}, \ldots, x_{t}\right]$ (therefore without the additional variable $\mathrm{x}$ ). Given $f \in$ $\mathcal{M}$, the map $\mathcal{P}_{\Phi}$ fully generates $\mathrm{f}$ if $f=\Phi\left(g_{1}, g_{2}, \ldots, g_{t}\right)$ with $g_{i} \in \mathcal{N} \cup \mathcal{K}$ for all $\mathrm{s}$ (obviously, $\mathcal{P}_{\Phi}\left(g_{1}, g_{2}, \ldots, g_{t}\right)=$ $(f, 0,0,0, \ldots))$.

A high number of fully generated polynomials was obtained by considering $\Phi=x_{1} x_{2}+\alpha x_{1}+\beta x_{2}+\gamma$ with $|\alpha|,|\beta| \leq 1$ and $0<|\gamma| \leq 3$. We have the following list of identities: 


$\begin{array}{lll}f_{1}^{2}+f_{1}-1=f_{5} & f_{8} f_{11}-f_{8}-1=f_{65} & f_{11} f_{36}-f_{11}+1=f_{105} \\ f_{1}^{2}+2 f_{1}-2=f_{6} & f_{11}^{2}-f_{11}-1=f_{68} & f_{24} f_{27}+f_{24}-1=f_{108} \\ f_{1} f_{6}+f_{6}+1=f_{29} & f_{7} f_{14}+f_{7}-1=f_{69} & f_{24} f_{27}+f_{27}-1=f_{109} \\ f_{1} f_{8}-f_{1}-1=f_{29} & f_{11} f_{14}+f_{11}-f_{14}-2=f_{73} & f_{6} f_{47}+f_{47}-1=f_{113} \\ f_{1} f_{8}-f_{1}+f_{8}-2=f_{31} & f_{8} f_{15}-f_{8}-1=f_{80} & f_{1} f_{92}+f_{1}+f_{92}+2=f_{115} \\ f_{1} f_{12}-f_{1}-1=f_{33} & f_{7} f_{18}+f_{18}+1=f_{81} & f_{8} f_{58}-f_{8}+1=f_{122} \\ f_{2}^{2}-f_{2}-1=f_{33} & f_{2} f_{31}+f_{31}+1=f_{82} & f_{30} f_{31}+f_{30}-f_{31}-2=f_{122} \\ f_{2}^{2}+f_{2}-1=f_{36} & f_{1} f_{48}+f_{1}-1=f_{83} & f_{8} f_{69}+f_{8}-f_{69}-2=f_{124} \\ f_{1} f_{19}-f_{1}+f_{19}-2=f_{44} & f_{10} f_{17}+f_{10}+1=f_{83} & f_{8} f_{91}-f_{8}+1=f_{133} \\ f_{1} f_{20}-f_{1}+f_{20}-2=f_{45} & f_{8} f_{23}-f_{23}-1=f_{86} & f_{29} f_{58}-f_{29}-1=f_{135} \\ f_{1} f_{24}-f_{1}+f_{24}-2=f_{46} & f_{8} f_{27}-f_{27}-1=f_{91} & f_{48} f_{49}+f_{49}-1=f_{141} \\ f_{6} f_{8}-f_{6}+f_{8}-2=f_{58} & f_{14} f_{19}+f_{19}+1=f_{91} & f_{14} f_{120}-f_{14}-1=f_{148} \\ f_{1} f_{30}+f_{30}+1=f_{59} & f_{6} f_{30}+f_{30}-1=f_{98} & f_{5} f_{122}-f_{5}-1=f_{150} \\ f_{7} f_{8}-f_{7}-1=f_{60} & f_{6} f_{31}+f_{6}+1=f_{98} & \\ f_{6} f_{12}+f_{12}-1=f_{62} & f_{8} f_{29}-f_{8}-f_{29}+2=f_{98} & \\ f_{8} f_{11}-f_{11}-1=f_{64} & f_{8} f_{29}-f_{8}+1=f_{99} & \end{array}$

The above identities show that some polynomials in $\mathcal{N}$ can be ruled out if we are interested in constructing a minimal set of "full" generators, that is, generators which are assumed to replace the $t$ variables of a suitable monic polynomial $\Phi\left(x_{1}, \ldots, x_{t}\right)$ with no additional variable $x$. Let $L$ and $R$ denote the subsets of polynomials $f_{i}$ that occur respectively at the left and at the right sides of these identities. In details, the corresponding subset of indices are

$$
\begin{gathered}
\hat{L}=\{1,2,5,6,7,8,10,11,12,14,15,17,18,19,20,23,24, \\
27,29,31,30,36,47,48,49,58,69,92,120,122\}, \\
\hat{R}=\{5,6,29,31,33,36,44,45,46,58,59,60,62,64,65,68, \\
\\
69,73,80,81,82,83,86,91,98,99,105,108, \\
\\
109,113,115,122,124,133,135,141,148,150\} .
\end{gathered}
$$

Although $L \cap R$ is not empty, the 9 polynomials in common are all generated by polynomials in $L \backslash R$. This is immediate to check in the case of $f_{5}, f_{6}, f_{29}, f_{31}, f_{36}, f_{69}$, and $f_{91}$, while $f_{58}=f_{6} f_{8}-f_{6}+f_{8}-2=\left(f_{1}^{2}+2 f_{1}-2\right) f_{8}-\left(f_{1}^{2}+2 f_{1}-2\right)+$ $f_{8}-2$, and similarly with $f_{122}$. Therefore, at this stage we can say that $(\mathcal{N} \backslash R) \cup\{1\}$ can be taken as a set of generators of $\mathcal{N}$. In order to reduce the number of generators we resort to a second placenta map whose related polynomial is $\Phi=x_{1} x_{2}+\alpha x_{1}+\beta x_{2}+\gamma x_{3}+\delta$ with $x_{3} \preceq x_{1},|\alpha|,|\beta|,|\gamma| \leq 1$ and $\delta \in\{-1,-2,-3\}$ (remarkably, positive coefficients of $\delta$ were occurring rarely in this experiment and we decided to exclude them, to speed up the computation). It turns out that $\mathcal{P}_{\Phi}$ generates a satisfactory number of polynomials in $\mathcal{N} \backslash R$. In the corresponding list, we write $(i, j, k, \alpha, \beta, \gamma, \delta) \rightarrow$ $q$ to indicate that $f_{q}$ is generated in particular by replacing $x_{1}, x_{2}, x_{3}$ with $f_{i}, f_{j}, f_{k}$ respectively.

$$
\begin{array}{ll}
(4,1,3,1,0,1,-2) \rightarrow 22 & (6,7,8,-1,1,1,-3) \rightarrow 57 \\
(8,1,1,1,-1,-1,-3) \rightarrow 30 & (7,12,14,0,1,-1,-2) \rightarrow 63 \\
(2,2,1,-1,1,1,-2) \rightarrow 35 & (10,11,3,0,0,1,-1) \rightarrow 66 \\
(12,1,2,0,0,1,-2) \rightarrow 35 & (20,3,4,-1,0,1,-1) \rightarrow 67 \\
(11,1,11,1,-1,1,-1) \rightarrow 37 & (14,8,2,-1,1,1,-1) \rightarrow 70 \\
(19,1,12,1,-1,-1,-3) \rightarrow 42 & (43,1,21,1,-1,-1,-3) \rightarrow 78 \\
(19,1,11,1,-1,-1,-1) \rightarrow 43 & (16,12,24,1,-1,-1,-1) \rightarrow \\
(12,2,8,-1,1,1,-1) \rightarrow 47 & (21,8,12,0,0,1,-1) \rightarrow 85 \\
(12,2,8,0,1,1,-2) \rightarrow 48 & (38,2,20,1,-1,1,-1) \rightarrow 87 \\
(22,1,25,1,-1,1,-3) \rightarrow 49 & (51,1,9,1,-1,-1,-1) \rightarrow 88 \\
(12,3,1,1,1,-1,-1) \rightarrow 51 & (37,3,20,1,-1,1,-1) \rightarrow 89 \\
(27,1,12,1,-1,1,-1) \rightarrow 52 & (20,13,7,1,0,1,-1) \rightarrow 90 \\
(26,1,25,1,-1,1,-3) \rightarrow 53 & (20,14,10,1,-1,1,-1) \rightarrow 92 \\
(14,2,14,1,-1,1,-1) \rightarrow 54 & (20,14,12,1,-1,1,-1) \rightarrow 93
\end{array}
$$

$$
\begin{aligned}
& (26,12,27,0,0,1,-1) \rightarrow 96 \\
& (31,5,8,-1,1,1,-1) \rightarrow 97 \\
& (30,8,1,0,-1,-1,-2) \rightarrow 100 \\
& (15,18,9,1,-1,1,-1) \rightarrow 101 \\
& (32,12,28,-1,1,-1,-1) \rightarrow 104 \\
& (25,27,17,0,0,-1,-1) \rightarrow 110 \\
& (77,2,40,1,-1,1,-1) \rightarrow 120 \\
& (30,37,28,1,-1,1,-1) \rightarrow 123 \\
& (65,14,21,1,0,-1,-2) \rightarrow 126 \\
& (52,24,1,0,0,-1,-1) \rightarrow 128 \\
& (72,14,23,1,-1,-1,-1) \rightarrow 129 \\
& (58,31,59,1,-1,-1,-1) \rightarrow 137
\end{aligned}
$$


As to the set of indices of $L^{\prime}$ and $R^{\prime}$ (using similar symbols as above) we have:

$$
\begin{aligned}
\hat{L}^{\prime}=\{ & 1,2,3, \ldots, 26,27,28,30,31,32,37,38,40,43,51,52, \\
& 58,59,65,72,77\}, \\
\hat{R}^{\prime}=\{ & 22,30,35,37,42,43,47,48,49,51,52,53,54,57,63,66, \\
& 67,70,78,84,85,87,88,89,90,92,93,96,97,100,101, \\
& 104,110,120,123,126,128,129,137\} .
\end{aligned}
$$

In the present case there are 5 polynomials in common and they are all generated by elements of $L^{\prime} \backslash R^{\prime}$. In conclusion, the set of polynomials we can rule out increases to $R \cup R^{\prime}$.

In order to further reduce the set of generators of $\mathcal{N}$ we should detect other suitable placenta maps. The many identities at our disposal can be helpful in finding such maps. For example-coming back to the first list-the two recursive ways of generating $f_{150}$ through the already obtained $f_{122}$ suggest to consider two further placenta maps, using the polynomials $x_{1} x_{2} x_{3}+x_{1} x_{3}-x_{1} x_{2}-3 x_{1}-1$ and $x_{1} x_{2} x_{3}-x_{1} x_{2}-1$. Actually, we already came across these maps when looking for general factors, but now the purpose is more specific and the support of algebra is crucial. For example, taking $\Phi=$ $x_{1} x_{2} x_{3}+\alpha x_{1} x_{2}+\beta$ we find two further identities:

$$
\begin{aligned}
f_{1} f_{19} f_{44}+f_{19} f_{44}-1 & =f_{139} \\
f_{8} f_{14} f_{40}+f_{8} f_{40}+1 & =f_{143}
\end{aligned}
$$

Moreover, by Proposition 3 we have $f_{2} f_{8} f_{15} f_{31}-1=f_{156}$. There could probably be many ways of obtaining even better results, but as our main purpose is the presentation of a general method, we stop here and draw the expected conclusion.

Proposition 5. Let $\mathcal{H}$ be defined as $\mathcal{N} \backslash\left(\mathcal{R} \cup \mathcal{R}^{\prime} \cup\left\{f_{139}\right.\right.$, $\left.\left.f_{143}, f_{156}\right\}\right)$. The set $\mathcal{N}$ is fully generated by polynomials in $\mathcal{H}$; the polynomials $\Phi$ giving rise to the related maps $\mathcal{P}_{\Phi}$ are either of the form $x_{1} x_{2}+\alpha x_{1}+\beta x_{2}+\gamma x_{3}+\delta$ or $x_{1} x_{2} x_{3}+\alpha x_{1} x_{2}+$ $\beta$, for some small coefficients $\alpha, \beta, \gamma, \delta$ whose absolute value does not exceed 3 in any case, together with $x_{1} x_{2} x_{3} x_{4}-1$.

Note that the cardinality of $\mathcal{H}$ is 80 . Note also that if we allow the presence of Kronecker polynomials in our computations, then we can easily generate also some polynomials of very small degree with the aid of other polynomials of likewise small degree.

The set $\mathcal{H}$ contains polynomials which we could regard as special. We are then led to conclude this section with a conjecture that, we hope, suggests a line of research in the future.

Conjecture 6. Every polynomial in $\mathcal{M}$ is fully generated by polynomials in $\mathcal{H}$.

Clearly, every other subset of generating polynomials possibly smaller than $\mathcal{H}$ is a good candidate for another conjecture of the same kind.

\section{Some families of Kronecker polynomials}

In this section we are going to express all the relevant polynomials in terms of suitable Chebyshev polynomials. This is motivated by observations recalled in the Introduction. We indeed find that several families of polynomials have simple expression in terms of Chebyshev polynomials.

Consider Chebyshev polynomials as defined in [7] for $n>0$ :

$$
T_{n}(x)=x^{n}+\sum_{k=1}^{\left\lfloor\frac{n}{2}\right\rfloor}(-1)^{k} \frac{n}{k}\left(\begin{array}{c}
n-k-1 \\
k-1
\end{array}\right) x^{n-2 k}
$$

and $T_{0}(x)=1$. Notice that these are the Chebyshev polynomials in $[-2,2]$ satisfying $T_{n}(2 \cos \theta)=2 \cos (n \theta)$.

Let $\mathcal{B}=\left(T_{0}(x), T_{1}(x), T_{2}(x), \ldots\right)$ be the ordered basis made up of these monic polynomials and let $m$ be the matrix of the change of basis from the standard basis $\mathcal{S}=$ $\left(1, x, x^{2}, x^{3}, \ldots\right)$ to $\mathcal{B}$.

The matrix $m$ is infinite, upper triangular with all 1's on the main diagonal. Its entries are

$$
\begin{aligned}
m_{0,0} & =1, \quad m_{0,2 j+1}=0, \quad j=0,1, \ldots, \\
m_{0,2 j} & =(-1)^{j} 2, \quad j=1,2, \ldots \\
m_{2 i, 2 j} & =(-1)^{i+j}\left(\begin{array}{c}
i+j-1 \\
2 i-1
\end{array}\right) \frac{j}{i}, \\
m_{2 i, 2 j+1} & =0, \quad i=1,2, \ldots, \quad j=0,1, \ldots, \\
m_{2 i+1,2 j} & =0, m_{2 i+1,2 j+1}=(-1)^{i+j}\left(\begin{array}{c}
i+j \\
2 i
\end{array}\right) \frac{2 j+1}{2 i+1}, \\
i & =0,1, \ldots, \quad j=0,1, \ldots,
\end{aligned}
$$

The inverse matrix $b=m^{-1}$ is also infinite and upper triangular and its entries are

$$
\begin{aligned}
b_{2 i, j} & =\frac{1+(-1)^{j}}{2}\left(\begin{array}{c}
j \\
\frac{j+2 i}{2}
\end{array}\right), i \geq 0, j \geq 0, \\
b_{2 i+1, j} & =\frac{1-(-1)^{j}}{2}\left(\begin{array}{c}
j \\
\frac{j-2 i-1}{2}
\end{array}\right), i \geq 0, j \geq 0 .
\end{aligned}
$$

In what follows we are going to examine some families of polynomials whose coordinates in the Chebyshev basis are particularly simple and regular. In general, for a family of polynomials we would like to ascertain whether they have all their roots in the critical interval $[-2,2]$ and if not, whether the span is "small" or not.

As mentioned in the Introduction, we are going to use the transformation $x=z+z^{-1}$, where $x \in \mathbb{R}$ and $z \in \mathbb{C}$. Notice that $x \in \mathbb{R}$ implies that either $z \in \mathbb{R}$, and so $|x| \geq 2$, or $z$ lies on the unit circle and $|x| \leq 2$.

Recall that for the Chebyshev polynomials of the first kind, this transformation gives, for $n>0$,

$$
T_{n}(x)=T_{n}\left(z+z^{-1}\right)=z^{n}+z^{-n} .
$$

Notation: Given a function $f(x)$, we shall write $\tilde{f}(z)=$ $f\left(z+z^{-1}\right)$. Obviously, $\tilde{T}_{0}(z)=1$.

For $k, s$ nonnegative integers, and an integer $n>1$, let $P_{k, n}^{(s)}(x)$ be a polynomial with Chebyshev coordinates

$$
[\underbrace{0, \ldots, 0}_{s}, \underbrace{1,0, \ldots, 0}_{k+1}, \underbrace{1,0, \ldots, 0}_{k+1}, \ldots, \underbrace{1,0, \ldots, 0}_{k+1}, 1]
$$

where $n$ is the number of 1's. 
Proposition 7. The polynomials $P_{k, n}^{(s)}(x)$, is of Kronecker type. Moreover, for fixed $k$ and $s$, as $n$ approaches $+\infty$, the largest root tends to 2 and the smallest to -2 .

Proof. We have

$$
\begin{aligned}
\tilde{P}_{k, n}^{(s)}(z) & =\tilde{T}_{s}(z)+\tilde{T}_{k+1+s}(z)+\cdots+\tilde{T}_{(n-1)(k+1)+s}(z) \\
& =\sum_{j=0}^{n-1}\left(z^{j(k+1)+s}+z^{-(j(k+1)+s)}\right) .
\end{aligned}
$$

Hence

$z^{(n-1)(k+1)+s} \tilde{P}_{k, n}^{(s)}(z)$

$=z^{(n-1)(k+1)+s}\left(z^{s}+z^{-s}+\cdots+z^{(n-1)(k+1)+s}+z^{-((n-1)(k+1)+s)}\right)$

$=1+z^{k+1}+\cdots+z^{(n-1)(k+1)}+z^{(n-1)(k+1)+2 s}+\cdots+z^{2(n-1)(k+1)+2 s}$

$=\left(1+z^{(n-1)(k+1)+2 s}\right)\left(1+z^{k+1}+\cdots+z^{(n-1)(k+1)}\right)$

$=\left(1+z^{(n-1)(k+1)+2 s)} \frac{z^{n(k+1)}-1}{z^{k+1}-1}\right.$.

Then the roots of $z^{(n-1)(k+1)+s} \tilde{P}_{k, n}^{(s)}(z)$ are all on the unit circle. So, translating back to $x, P_{k, n}^{(s)}(x)$ is hyperbolic, its roots are in $[-2,2]$ and is therefore of Kronecker type.

Fixing $k$ and $s$, as $n$ approaches $+\infty$, the roots become dense in the unit circle and so the largest root of $P_{k, n}^{(s)}(x)$ tends to 2 and the smallest to -2 .

Proposition 8. Let $A_{n}(x)$ be a degree $n$ polynomial with Chebyshev coordinates

$$
[2,2,2, \ldots, 2,2,1],
$$

$B_{2 n}(x)$ a polynomial of even degree $2 n$ with Chebyshev coordinates

$$
[2,1,2,1, \ldots, 2,1,1]
$$

and $B_{2 n+1}(x)$, of odd degree, with Chebyshev coordinates

$$
[1,2,1,2,1, \ldots, 2,1,1] \text {. }
$$

Then $A_{n}(x), B_{2 n}(x)$, and $B_{2 n+1}(x)$ are all of Kronecker type and the largest root tends to 2 and the smallest to -2 as the degree increases.

Proof. We have

$$
\begin{aligned}
\tilde{A}_{n}(z) & =2 \tilde{T}_{0}(z)+2 \tilde{T}_{1}(z)+2 \tilde{T}_{2}(z)+\cdots+2 \tilde{T}_{n-1}(z)+\tilde{T}_{n}(z) \\
& =2\left(1+\sum_{j=1}^{n-1}\left(z^{j}+z^{-j}\right)\right)+z^{n}+z^{-n} .
\end{aligned}
$$

Hence

$$
\begin{aligned}
z^{n} \tilde{A}_{n}(z) & =2\left(1+z+z^{2}+\cdots+z^{2 n-1}\right)+z^{2 n}-1 \\
& =2 \frac{z^{2 n}-1}{z-1}+z^{2 n}-1=\left(z^{2 n}-1\right) \frac{z+1}{z-1},
\end{aligned}
$$

which again has all roots on the unit circle. It follows that $A_{n}(x)$ is a Kronecker polynomial.
Analogously,

$$
\begin{aligned}
\tilde{B}_{2 n}(z)= & 2 \tilde{T}_{0}(z)+\tilde{T}_{1}(z)+2 \tilde{T}_{2}(z)+\tilde{T}_{3}(z)+\cdots+2 \tilde{T}_{2 n-2}(z) \\
& +\tilde{T}_{2 n-1}(z)+\tilde{T}_{2 n}(z) \\
= & 2\left(1+\sum_{j=1}^{n-1}\left(z^{2 j}+z^{-2 j}\right)\right)+\sum_{j=1}^{n}\left(z^{2 j-1}+z^{-(2 j-1)}\right) \\
& +z^{2 n}+z^{-2 n} .
\end{aligned}
$$

and so

$$
\begin{aligned}
& z^{2 n} \tilde{B}_{2 n}(z) \\
& =2\left(1+z^{2}+\cdots+z^{4 n-2}\right)+z\left(1+z^{2}+\cdots+z^{4 n-2}\right)+z^{4 n}-1 \\
& =(2+z) \frac{z^{4 n}-1}{z^{2}-1}+z^{4 n}-1=\left(z^{4 n}-1\right) \frac{z^{2}+z+1}{z^{2}-1} .
\end{aligned}
$$

Hence $B_{2 n}(x)$ is a further family of Kronecker polynomials.

The case $B_{2 n+1}(x)$, with Chebyshev coordinates

$$
[1,2,1,2,1, \ldots, 2,1,1]
$$

is completely analogous.

Also in these cases the largest root tends to 2 and the smallest to -2 as the degree increases.

\section{Relations with Pisot's and Salem's numbers}

Let's recall the following definitions.

Definition 9. A Pisot number is a real algebraic number greater than 1 all of whose conjugate, except itself, lie inside the unit circle $|z|<1$.

Definition 10. A Salem number is a real algebraic number $\tau$ greater than 1 of degree at least 4 , conjugate to its inverse $\tau^{-1}$, all of whose conjugates, excluding $\tau$ and $\tau^{-1}$, lie on the unit circle $|z|=1$.

Let $P_{2 n+k-1}(x)$ be the family of polynomials of degree $2 n+k-1$ and coordinates

$$
[-1,0,-1,0, \ldots,-1, \underbrace{0, \ldots, 0}_{k}, 1],
$$

where $n \geq 1$ is the number of -1 's and $k$ a nonnegative integer.

$$
\begin{aligned}
\tilde{P}_{2 n+k-1}(z)= & -\tilde{T}_{0}(z)-\tilde{T}_{2}(z)-\tilde{T}_{4}(z)-\cdots-\tilde{T}_{2 n-2}(z) \\
& +\tilde{T}_{2 n+k-1}(z) \\
= & -\left(1+z^{2}+z^{-2}+z^{4}+z^{-4}+\cdots+z^{2 n-2}\right. \\
& \left.+z^{-(2 n-2)}\right)+z^{2 n+k-1}+z^{-(2 n+k-1)}
\end{aligned}
$$

and so

$$
\begin{aligned}
& z^{2 n+k-1}\left(z^{2}-1\right) \tilde{P}_{2 n+k-1}(z) \\
& =\left[-\left(z^{k+1}+z^{k+3}+\cdots+z^{4 n+k-3}\right)+1+z^{4 n+2 k-2}\right]\left(z^{2}-1\right) \\
& =z^{4 n+2 k}-z^{4 n+2 k-2}-z^{4 n+k-1}+z^{k+1}+z^{2}-1 \\
& =z^{4 n+k-1}\left(z^{k+1}-z^{k-1}-1\right)+z^{k+1}+z^{2}-1 .
\end{aligned}
$$


When $k=0$ this last step can be rewritten as $z^{4 n-2}\left(z^{2}-z-1\right)+z^{2}+z-1$.

We now observe that

Proposition 11. For $k \geq 1$ the polynomials $z^{k+1}-z^{k-1}-1$ and $z^{k+1}+z^{2}-1$ have equal absolute value on the unit circle. The same happens for $z^{2}-z-1$ and $z^{2}+z-1$ (case $k=0$ ).

Proof. Let $k \geq 1$ and set $z^{k+1}-z^{k-1}-1=f(z)$ and $z^{k+1}+$ $z^{2}-1=g(z)$, we notice that $g(z)=-z^{k+1} f\left(z^{-1}\right)$. For $|z|=$ 1 we have $f\left(z^{-1}\right)=f(\bar{z})$. Hence for $|z|=1$ we have

$$
|g(z)|=\left|z^{k+1}\right| f\left(z^{-1}\right)|=1| f(\bar{z})|=| \overline{f(z)}|=| f(z) \mid .
$$

A similar argument holds for the two polynomials $z^{2}-z-$ 1 and $z^{2}+z-1$.

In general, given two polynomials $f(x), g(x)$, where $g(z)= \pm z^{\operatorname{deg} f} f\left(z^{-1}\right), f(x)$ and $g(x)$ have the same absolute value on the unit circle.

We then have

Theorem 12. For $k=0,1,2,3,5,7$, the polynomials $P_{2 n+k-1}(x)$ are hyperbolic with all roots in the interval $[-2,2]$ except for one $x_{M}>2$, moreover $\lim _{n \rightarrow \infty} x_{M}=z_{0}+z_{0}^{-1}$, where $z_{0}$ is the largest real root of the polynomial $z^{k+1}$ $z^{k-1}-1\left(z^{2}-z-1\right.$ for $\left.k=0\right)$, while the smallest root $x_{m}$ approaches -2 .

\section{Proof. Let}

$$
\begin{aligned}
R(z) & =z^{2 n+k-1}\left(z^{2}-1\right) \tilde{P}_{2 n+k-1}(z) \\
& =\left\{\begin{array}{ll}
z^{4 n-2}\left(z^{2}-z-1\right)+z^{2}+z-1, & k=0 \\
z^{4 n+k-1}\left(z^{k+1}-z^{k-1}-1\right)+z^{k+1}+z^{2}-1, & k \geq 1
\end{array} .\right.
\end{aligned}
$$

For $k=0, z^{2}-z-1$ is the minimal polynomial of a Pisot number. Since Proposition 11 guarantees that $\mid z^{2}-$ $z-1|=| z^{2}+z-1 \mid$ on the unit circle, then a standard result of Salem, [12], Chapter 3, Section 4, (see also [10, 11] and [14]) implies that, for large enough $n, R(z)$ is the minimal polynomial of a Salem number, possibly multiplied by some cyclotomic polynomials.

It is then clear that the largest root of $R(z)$ approaches, as $n$ goes to infinity, the largest root $z_{0}(>1)$ of $z^{2}-z-1$, another root approaches $\frac{1}{z_{0}}$, while all the other roots are on the unit circle, and, as $n$ goes to infinity, they become dense there. Thus, translating back to $x$, all roots $x$ are real, the smallest root tends to -2 and the largest to $z_{0}+z_{0}^{-1}$.

For $k=2, z^{k+1}-z^{k-1}-1=z^{3}-z-1$ is the minimal polynomial of a Pisot number and we can repeat the same argument as above.

For odd $k$, setting $k=2 h+1$, the polynomial $z^{k+1}-$ $z^{k-1}-1=z^{2 h+2}-z^{2 h}-1$ cannot be a minimal polynomial of a Pisot number, so we set $z^{2}=y$ and obtain $y^{h+1}-y^{h}-$ 1. $R(z)$, as a function of $y$, is $y^{2 n+h}\left(y^{h+1}-y^{h}-1\right)+y^{h+1}+$ $y-1$. For $h=0,1,2,3$ these are minimal polynomials of a Pisot number so an argument similar to the even cases $k=0,2$ shows that $y^{2 n+h}\left(y^{h+1}-y^{h}-1\right)+y^{h+1}+y-1$ is a minimal polynomial of a Salem number. Hence $R(z)$ has pairs of opposite roots on the unit circle except for four on the real axis, the smallest less than -1 , the largest greater than 1 and two more in the interval $(-1,1)$. As $n$ goes to infinity the largest root tends to the largest root of $z^{2 h+2}-$ $z^{2 h}-1$. Thus, translating back to $x$, all roots $x$ are real, the greatest tends to $z_{0}+z_{0}^{-1}$ (and the smallest to $\left.-\left(z_{0}+z_{0}^{-1}\right)\right)$.

Remark 13. For $k$ even and greater than 2 and for $k$ odd and greater than 7 unfortunately $z^{k+1}-z^{k-1}-1$ is not the minimal polynomial of a Pisot number. For even $k$, besides having a real root greater than 1 , it also has two complex conjugates roots outside of the unit circle. For $k$ odd, after setting $z^{2}=y$, we have an analogous situation. Thus, Salem construction in theory is not possible. However, although the polynomials of the family are not hyperbolic, for even $k>2$, they still have a largest real root that approaches the largest real root of $z^{k+1}-z^{k-1}-1$ and the smallest root approaches -2 while, for odd $k$, the largest and smallest root, that are opposite, approach the largest and smallest, respectively, of $z^{k+1}-z^{k-1}-1$.

Actually, these polynomials are almost hyperbolic, in the sense that, for even $k$, they have only a pair of complex conjugate roots, and for odd $k$ only two opposite pairs of complex conjugate roots.

\subsection{A two-parameter family}

In this section, we consider the family of polynomials $P_{\left(h_{1}, h_{2}\right)}^{(n)}(x)$ depending on two integer parameters $h_{1}, h_{2}$ with $1<h_{1} \leq h_{2}$, with Chebyshev coordinates

$$
[1, \underbrace{-h_{1}, h_{2},-h_{1}, h_{2}, \ldots,-h_{1}, h_{2}}_{2 n}, 1]
$$

of degree $2 n+1$ in $x$. We want to rewrite this polynomial in a suitable way:

$$
\begin{aligned}
\tilde{P}_{\left(h_{1}, h_{2}\right)}^{(n)}(z)= & \tilde{T}_{0}(z)-h_{1} \tilde{T}_{1}(z)+h_{2} \tilde{T}_{2}(z)-\cdots-h_{1} \tilde{T}_{2 n-1}(z) \\
& +h_{2} \tilde{T}_{2 n}(z)+\tilde{T}_{2 n+1}(z) \\
= & 1-h_{1}\left(z+z^{-1}\right)+h_{2}\left(z^{2}+z^{-2}\right) \\
& +\cdots+\left(z^{2 n+1}+z^{-(2 n+1)}\right)
\end{aligned}
$$

Add and subtract $h_{2}$ and multiply by $z^{2 n+1}\left(z^{2}-1\right)$ :

$$
\begin{aligned}
& z^{2 n+1}\left(z^{2}-1\right) \tilde{P}_{\left(h_{1}, h_{2}\right)}^{(n)}(z) \\
& =z^{2 n+1}\left\{z^{2 n+1}\left[z^{2}+h_{2} z-\left(h_{1}+1\right)\right]-\frac{h_{2}-1}{2}\left(z^{2}-1\right)\right\} \\
& -\frac{h_{2}-1}{2} z^{2 n+1}\left(z^{2}-1\right)+\left(1+h_{1}\right) z^{2}-h_{2} z-1
\end{aligned}
$$

which we rewrite as

$$
z^{2 n+1}\left(z^{2}-1\right) \tilde{P}_{\left(h_{1}, h_{2}\right)}^{(n)}(z)=z^{2 n+1} Q(z)-z^{2 n+3} Q\left(z^{-1}\right)
$$

where $Q(z)=z^{2 n+1}\left[z^{2}+h_{2} z-\left(h_{1}+1\right)\right]-\frac{h_{2}-1}{2}\left(z^{2}-1\right)$. If $Q(z)$ is the minimal polynomial of a Pisot number then, by 
Salem construction, $z^{2 n+1} \tilde{P}_{\left(h_{1}, h_{2}\right)}^{(n)}(z)$ is, (for sufficiently large $n$ ) the minimal polynomial of a Salem number, possibly multiplied by some cyclotomic polynomials.

If $h_{2}$ is even $Q(z)$ does not have integer coefficients and so it is not the minimal polynomial of a Pisot number. Given that $z^{2 n+1} \widetilde{P}_{\left(h_{1}, h_{2}\right)}^{(n)}(z)$ has integer coefficients, as long as $Q(z)$ has a root greater than 1 in absolute value and all the others are inside the unit disc, then the Salem construction still works.

Set $U(z)=z^{2}+h_{2} z-\left(h_{1}+1\right)$. Recall that $0<h_{1} \leq h_{2}$, and since $U(-1)=-h_{1}-h_{2}<0$ while $U(1)=h_{2}-h_{1} \geq$ 0 , we know that $U(z)$ has a real root $\alpha<-1$ and the other in $(-1,1]$. So $\alpha$ is the negative of a Pisot number. In the case $h_{1}=h_{2}$ we have $U(z)=(z-1)\left(z+h_{1}+1\right)$.

Lemma 14. On $|z|=1$,

$$
\left|\frac{h_{2}-1}{2}\left(z^{2}-1\right)\right| \leq|U(z)|
$$

Proof. For $z=a+i b$ on the unit circle we have

$$
\begin{aligned}
& |U(z)|^{2}-\left|\frac{h_{2}-1}{2}\left(z^{2}-1\right)\right|^{2} \\
& =\left(h_{1}-h_{2} a\right)^{2}+\left(4 h_{1}+2 h_{2}+3\right) b^{2} \geq 0 .
\end{aligned}
$$

Theorem 15. For every pair of integers $\left(h_{1}, h_{2}\right)$, with $0<h_{1} \leq h_{2}$, the polynomials of the family with Chebyshev coordinates

$$
\left[1,-h_{1}, h_{2},-h_{1}, h_{2}, \ldots,-h_{1}, h_{2}, 1\right]
$$

are hyperbolic with all roots in the interval $[-2,2]$ except for one $x_{m}<-2$, moreover $\lim _{n \rightarrow \infty} x_{m}=z_{0}+z_{0}^{-1}$, where $z_{0}$ is the smallest real root of the polynomial $z^{2}+h_{2} z-\left(h_{1}+1\right)$, while the largest root $x_{M}$ approaches 2.

Proof. A standard argument, based on Rouchés theorem and using the previous lemma, shows that $Q(z)$ in $(4-3)$ has a real root less than -1 and all the others with $|z|<1$, in other words it corresponds to the negative of a Pisot number (in an extended sense if $h_{2}$ is even as the coefficients may not be integers). Therefore $z^{2 n+1}\left(z^{2}-1\right) \tilde{P}_{\left(h_{1}, h_{2}\right)}^{(n)}(z)$ is the minimal polynomial of the negative of a Salem number, possibly multiplied by some cyclotomic polynomials. From (4-2) one can deduce that as $n$ goes to $+\infty$ the Salem number approaches the smallest root $z_{0}$ of $z^{2}+h_{2} z-\left(h_{1}+1\right)$ and the other roots become dense on the unit circle. Translating back to $x$ we see that $P_{\left(h_{1}, h_{2}\right)}^{(n)}(x)$ is hyperbolic, with one root less than -2 and all the other in $[-2,2]$, and as $n$ goes to $+\infty$ the smallest root tends to $x_{m}=z_{0}+\frac{1}{z_{0}}$ and the largest $x_{M}$ tends to 2 .

Remark 16. It can be shown that the limit of the span, namely $2-z_{0}-z_{0}^{-1}$, is the largest root of the resultant with respect to $z$ of $z^{2}+h_{2} z-h_{1}-1$ and $\left(x-\left(2-z-z^{-1}\right)\right) z$ :

$$
-\left(h_{1}+1\right) x^{2}+\left(4\left(h_{1}+1\right)+h_{1} h_{2}\right) x+\left(h_{2}-h_{1}\right)^{2}
$$

Remark 17. Elementary calculations also show that $\lim _{n \rightarrow \infty} x_{m}$ is the negative root of the polynomial

$$
\left(h_{1}+1\right) x^{2}+h_{1} h_{2} x-\left[h_{2}^{2}+\left(h_{1}+2\right)^{2}\right] \text {. }
$$

\subsection{Case of three parameters}

Consider the family of polynomials $P_{\left(h_{1}, h_{2}, h_{3}\right)}^{(n)}(x)$ of degree $6 n+1$ depending on the integer parameters $h_{1}, h_{2}, h_{3}$, with $1<h_{1} \leq h_{2} \leq h_{3}$, excluding the case $h_{1}=h_{2}=h_{3}$ which is included in the previous section, and Chebyshev coordinates

$$
\left[1,-h_{1}, h_{2},-h_{3}, h_{1},-h_{2}, h_{3}, \ldots,-h_{1}, h_{2},-h_{3}, h,-h_{2}, h_{3}, 1\right] .
$$

We have

$$
\begin{aligned}
z^{6 n+1}\left(z^{3}+1\right) \tilde{P}_{\left(h_{1}, h_{2}, h_{3}\right)}^{(n)}(z) \\
=z^{6 n+1}\left[z^{6 n+1}\left(z^{3}+h_{3} z^{2}-h_{2} z+h_{1}+1\right)\right. \\
\left.\quad+1-h_{3}+\left(h_{2}-h_{1}\right) z+\left(h_{2}-h_{1}\right) z^{2}+\left(1-h_{3}\right) z^{3}\right] \\
\quad+\left(h_{1}+1\right) z^{3}-h_{2} z^{2}+h_{3} z+1 .
\end{aligned}
$$

Setting

$$
\begin{aligned}
Q(z)= & z^{6 n+1}\left(z^{3}+h_{3} z^{2}-h_{2} z+h_{1}+1\right) \\
& +\frac{1-h_{3}+\left(h_{2}-h_{1}\right) z+\left(h_{2}-h_{1}\right) z^{2}+\left(1-h_{3}\right) z^{3}}{2}
\end{aligned}
$$

one gets

$$
z^{6 n+1}\left(z^{3}+1\right) \tilde{P}_{\left(h_{1}, h_{2}, h_{3}\right)}^{(n)}(z)=z^{6 n+1} Q(z)+z^{6 n+4} Q\left(z^{-1}\right) .
$$

Lemma 18. The polynomial

$$
U(z)=z^{3}+h_{3} z^{2}-h_{2} z+h_{1}+1
$$

has a real root $\alpha<-1$ and the others in the unit disc, so $\alpha$ is the negative of a Pisot number.

Proof. Notice that the polynomial $U(z)$ computed at $-\left(h_{1}+1\right)$ is certainly nonnegative:

$$
U\left(-\left(h_{1}+1\right)\right)=\left(h_{1}+1\right)\left(-h_{1}^{2}-2 h_{1}+h_{3} h_{1}+h_{3}+h_{2}\right) \geq 0
$$

then a real root $\alpha$ must be smaller than $-\left(h_{1}+1\right)$.

Let $\beta, \gamma$ the other two roots. Then $|\alpha \beta \gamma|=h_{1}+1$ so that

$$
|\beta \gamma|=\frac{h_{1}+1}{|\alpha|}<1 .
$$

Suppose that $\gamma=\bar{\beta}$, hence $|\beta|^{2}=\frac{h_{1}+1}{\alpha \mid}<1$. Next, suppose $\beta$ and $\gamma$ are real. From (4-7), it follows that one of the two, say $\beta$, satisfies $-1<\beta<1$. Now, since $U(-1)=$ $h_{1}+h_{2}+h_{3}>0$ and $U(1)=2+h_{1}-h_{2}+h_{3}>0, \quad \gamma \quad$ as well must lie between -1 and 1 . Therefore, in all cases, $\beta$ and $\gamma$ are in the unit disk. 
Lemma 19. Set

$$
U(z)=z^{3}+h_{3} z^{2}-h_{2} z+h_{1}+1
$$

and

$$
V(z)=\frac{1-h_{3}+\left(h_{2}-h_{1}\right) z+\left(h_{2}-h_{1}\right) z^{2}+\left(1-h_{3}\right) z^{3}}{2},
$$

then on the unit circle we have $|U(z)|^{2} \geq|V(z)|^{2}$.

Proof. Setting $z=x+i y$, on the unit circle we have

$$
\begin{aligned}
& |U(z)|^{2}=a(x)=\left(x^{3}-3 x\left(1-x^{2}\right)+h_{3}\left(2 x^{2}-1\right)\right. \\
& \left.-h_{2} x+h_{1}+1\right)^{2}+\left(1-x^{2}\right)\left(4 x^{2}+2 h_{3} x-h_{2}-1\right)^{2}
\end{aligned}
$$

and

$$
\begin{aligned}
& |V(z)|^{2}=b(x) \\
& =\frac{1}{4}\left(\left(\left(h_{3}-1\right)\left(4 x^{3}-3 x+1\right)+\left(h_{1}-h_{2}\right)\left(2 x^{2}+x-1\right)\right)^{2}\right. \\
& \left.+\left(1-x^{2}\right)\left(\left(h_{3}-1\right)\left(4 x^{2}-1\right)+\left(h_{1}-h_{2}\right)(2 x+1)\right)^{2}\right)
\end{aligned}
$$

Moreover, setting $f(x)=a(x)-b(x)$ we must show that $f(x) \geq 0$ for $-1 \leq x \leq 1$. It turns out that $f(x)$ is a third degree polynomial function, precisely

$$
\begin{aligned}
f(x)= & \left(-2 h_{3}^{2}+4 h_{3}+8 h_{1}+6\right) x^{3} \\
& +2\left(h_{2}\left(h_{3}-3\right)+2 h_{3}+h_{1}\left(h_{3}+1\right)\right) x^{2} \\
& +\frac{1}{2}\left(-h_{1}^{2}-2\left(h_{2}+h_{3}+5\right) h_{1}-h_{2}^{2}+3 h_{3}^{2}-2 h_{3}\right. \\
& \left.-2 h_{2}\left(h_{3}+3\right)-9\right) x \\
& +\frac{1}{2}\left(h_{1}^{2}+2\left(h_{2}-h_{3}+1\right) h_{1}+h_{2}^{2}+h_{3}^{2}-2 h_{2}\left(h_{3}-3\right)\right. \\
& \left.-2 h_{3}+3\right) .
\end{aligned}
$$

Reordering the expression according to the powers of the parameters $h_{i}$, we get

$$
\begin{aligned}
f(x)= & \frac{1}{2}\left(12 x^{3}-9 x+3\right)+\left(8 x^{3}+2 x^{2}-5 x+1\right) h_{1} \\
& +\left(-6 x^{2}-3 x+3\right) h_{2} \\
& +\left(4 x^{3}+4 x^{2}-x-1\right) h_{3}+(-x+1) h_{1} h_{2} \\
& +\left(2 x^{2}-x-1\right) h_{1} h_{3} \\
& +\left(2 x^{2}-x-1\right) h_{2} h_{3}+\frac{1}{2}(-x+1) h_{1}^{2}+\frac{1}{2}(-x+1) h_{2}^{2} \\
& +\frac{1}{2}\left(-4 x^{3}+3 x+1\right) h_{3}^{2} .
\end{aligned}
$$

Now consider $2 f(x)$. One has:

$$
\begin{aligned}
2 f(x)= & (1-x)\left(h_{1}+h_{2}-(1+2 x) h_{3}\right)^{2}+A_{0}(x)+A_{1}(x) h_{1} \\
& +A_{2}(x) h_{2}+A_{3}(x) h_{3}
\end{aligned}
$$

where we set $A_{0}(x)=3-9 x+12 x^{3}, A_{1}(x)=2(1-5 x+$ $\left.2 x^{2}+8 x^{3}\right), \quad A_{2}(x)=2\left(3-3 x-6 x^{2}\right), \quad A_{3}(x)=-2(1-x-$ $\left.2 x^{2}\right)(1+2 x)$. Since in the interval $\left[-1,-\frac{1}{2}\right]$ one has $A_{i}(x) \geq$ $0, i=0,1,2,3$, in this same interval $2 f \geq 0$. One cannot say the same thing in the interval $\left[-\frac{1}{2}, 1\right]$. The structure of the functions $A_{i}(x)$ suggests to introduce in the interval $\left[-\frac{1}{2}, 1\right]$ an auxiliary function $\alpha(x)$ and to decompose $2 f$ in the following fashion as a sum of positive quantities:

$$
\begin{aligned}
2 f= & (1-x)\left(h_{1}+h_{2}-(1+2 x) h_{3}+\alpha(x)\right)^{2} \\
& +R_{0}(x)+R_{1}(x) h_{1}+R_{2}(x) h_{2}+R_{3}(x) h_{3}
\end{aligned}
$$

where we set $R_{0}(x)=A_{0}(x)-(1-x) \alpha(x)^{2}, R_{1}(x)=A_{1}(x)$ $-2(1-x) \alpha(x), R_{2}(x)=A_{2}(x)-2(1-x) \alpha(x), R_{3}(x)=A_{3}(x)$ $+2(1-x)(1+2 x) \alpha(x)$.

Our task is to decompose the interval $\left[-\frac{1}{2}, 1\right]$ in subintervals where for each subinterval we choose a suitable $\alpha(x)$ such that the sum

$$
R(x)=R_{0}(x)+R_{1}(x) h_{1}+R_{2}(x) h_{2}+R_{3}(x) h_{3}
$$

is greater than or equal to zero.

In the interval $\left[-\frac{1}{2}, 0\right]$, by choosing $\alpha(x)=1-2 x$, we have $R_{i}(x) \geq 0, i=0,1,2,3$ and therefore $R(x) \geq 0$.

In the interval $\left[0, \frac{1}{7}\right]$, we choose $\alpha(x)=2-4 x$, and we have $R_{0}(x) \leq 0, R_{1}(x)<0, R_{2}(x)>0, R_{3}(x)>0$ so that

$$
\begin{aligned}
R(x) & =R_{0}(x)+R_{1}(x) h_{1}+R_{2}(x) h_{2}+R_{3}(x) h_{3} \\
& \geq\left(R_{0}(x)+R_{1}(x)+R_{2}(x)+R_{3}(x)\right) h_{1}
\end{aligned}
$$

It follows that $R(x) \geq 0$ since in $\left[0, \frac{1}{7}\right]$ the function $R_{0}(x)+$ $R_{1}(x)+R_{2}(x)+R_{3}(x)$ is nonnegative.

In the interval $\left[\frac{1}{7}, \frac{1}{4}\right]$, we choose again $\alpha(x)=2-4 x$. Now we have $R_{0}(x)>0, R_{1}(x)<0, R_{2}(x)>0, R_{3}(x)>0$, and $R_{1}(x)+R_{2}(x)+R_{3}(x) \geq 0$. Hence

$$
\begin{aligned}
R(x) & =R_{0}(x)+R_{1}(x) h_{1}+R_{2}(x) h_{2}+R_{3}(x) h_{3} \\
& \geq R_{0}(x)+\left(R_{1}(x)+R_{2}(x)+R_{3}(x)\right) h_{1} \geq 0 .
\end{aligned}
$$

Next, in the interval $\left[\frac{1}{4}, \frac{1}{2}\right]$, we choose $\alpha(x)=3-6 x$, and we have $R_{0}(x) \leq 0, R_{1}(x) \leq 0, R_{2}(x) \geq 0, R_{3}(x)>0$

$$
\begin{aligned}
R(x) & =R_{0}(x)+R_{1}(x) h_{1}+R_{2}(x) h_{2}+R_{3}(x) h_{3} \\
& \geq\left(R_{0}(x)+R_{1}(x)+R_{2}(x)+R_{3}(x)\right) h_{1} \geq 0
\end{aligned}
$$

since, in $\left[\frac{1}{4}, \frac{1}{2}\right], R_{0}(x)+R_{1}(x)+R_{2}(x)+R_{3}(x) \geq 0$.

Finally, in the interval $\left[\frac{1}{2}, 1\right]$, by choosing $\alpha(x)=$ $-3+6 x$, we have $R_{0}(x) \geq 0, R_{1}(x)>0, R_{2}(x) \leq 0, R_{3}(x) \geq$ $0, R_{2}(x)+R_{3}(x) \geq 0$.

$$
\begin{aligned}
R(x) & =R_{0}(x)+R_{1}(x) h_{1}+R_{2}(x) h_{2}+R_{3}(x) h_{3} \\
& \geq R_{0}(x)+R_{1}(x) h_{1}+\left(R_{2}(x)+R_{3}(x)\right) h_{2} \geq 0 .
\end{aligned}
$$


Lemma 18 and Lemma 19 and Rouchés Theorem imply that $Q(z)$ has a real root $<-1$ and the others in the unit disc. Therefore by mimicking the case of two parameters, we have

Theorem 20. For every triple of integers $\left(h_{1}, h_{2}, h_{3}\right)$, with $0<h_{1} \leq h_{2} \leq h_{3}$, the polynomials $P_{\left(h_{1}, h_{2}, h_{3}\right)}^{(n)}(x)$ of the family with Chebyshev coordinates

$$
\left[1,-h_{1}, h_{2},-h_{3}, h_{1},-h_{2}, h_{3}, \ldots,-h_{1}, h_{2},-h_{3}, h_{1},-h_{2}, h_{3}, 1\right]
$$

are hyperbolic, with all roots in the interval $[-2,2]$, except for one, which we denote $x_{m}$ and which is less than -2; moreover $\lim _{n \rightarrow \infty} x_{m}=z_{0}+z_{0}^{-1}$, where $z_{0}$ is the smallest real root of the polynomial $z^{3}+h_{3} z^{2}-h_{2} z+h_{1}+1$, while the largest root $x_{M}$ approaches 2 .

Remark 21. It can be shown that the limit of the span, which is $2-z_{0}-z_{0}^{-1}$, is the largest root of the resultant with respect to $z$ of $z^{3}+h_{3} z^{2}-h_{2} z+h_{1}+1$ and $\left(x-\left(2-z-z^{-1}\right)\right) z$ :

$$
\begin{aligned}
& -\left(h_{1}+1\right) x^{3}+\left[6\left(h_{1}+1\right)-h_{2}+h_{1} h_{3}+h_{3}\right] x^{2} \\
& -\left[9\left(h_{1}+1\right)-h_{1} h_{2}-h_{2}-h_{2} h_{3}+h_{3}-4\left(h_{2}-h_{1} h_{3}-h_{3}\right)\right] x \\
& +\left(h_{1}-h_{2}+h_{3}+2\right)^{2}
\end{aligned}
$$

Remark 22. Elementary calculations also show that $\lim _{n \rightarrow \infty} x_{m}$ is the negative root of the polynomial

$$
\begin{array}{r}
\left(h_{1}+1\right) x^{3}+\left(\left(h_{1}+1\right) h_{3}-h_{2}\right) x^{2} \\
-\left(\left(h_{1}+1\right)\left(h_{2}+3\right)+\left(h_{2}-1\right) h_{3}\right) x \\
+\left(h_{2}+1\right)^{2}+\left(h_{1}-h_{3}+1\right)^{2}
\end{array}
$$

In conclusion, using the methods we illustrated in the article it is relatively easy to obtain nonmonic polynomials that are hyperbolic, irreducible with small span. Although we found no new monic polynomials satisfying these conditions, this seems to confirm our feeling for the scarcity of such polynomials in high degree.

\begin{tabular}{|c|c|c|c|c|c|c|c|c|}
\hline (1) & -1 & -3 & & & & & & \\
\hline (2) & -1 & -3 & 1 & & & & & \\
\hline (3) & 0 & -4 & -2 & & & & & \\
\hline (4) & 0 & -4 & -1 & & & & & \\
\hline (5) & -2 & -4 & 5 & 5 & & & & \\
\hline (6) & -2 & -3 & 4 & 1 & & & & \\
\hline (7) & -2 & -3 & 5 & 1 & & & & \\
\hline (8) & -2 & -2 & 3 & 1 & & & & \\
\hline (9) & -1 & -4 & 0 & 1 & & & & \\
\hline (10) & -1 & -4 & 1 & 2 & & & & \\
\hline (11) & -1 & -4 & 2 & 3 & & & & \\
\hline (12) & -1 & -3 & 1 & 1 & & & & \\
\hline (13) & 0 & -5 & -1 & 4 & & & & \\
\hline (14) & 0 & -4 & -1 & 1 & & & & \\
\hline (15) & -2 & -4 & 6 & 4 & -2 & & & \\
\hline (16) & -2 & -4 & 6 & 4 & -1 & & & \\
\hline (17) & -2 & -4 & 7 & 4 & -5 & & & \\
\hline (18) & -2 & -3 & 4 & 2 & -1 & & & \\
\hline (19) & -2 & -3 & 5 & 1 & -1 & & & \\
\hline (20) & -2 & -3 & 6 & 0 & -1 & & & \\
\hline (21) & -1 & -5 & 2 & 5 & -1 & & & \\
\hline (22) & -1 & -5 & 3 & 5 & -2 & & & \\
\hline (23) & -1 & -5 & 3 & 6 & -1 & & & \\
\hline (24) & -1 & -5 & 4 & 5 & -3 & & & \\
\hline (25) & -1 & -5 & 5 & 4 & -3 & & & \\
\hline (26) & 0 & -6 & 0 & 8 & -1 & & & \\
\hline (27) & 0 & -5 & -1 & 5 & 1 & & & \\
\hline (28) & 0 & -5 & 0 & 4 & -1 & & & \\
\hline (29) & -3 & -3 & 11 & 3 & -9 & -1 & & \\
\hline (30) & -3 & -2 & 9 & 0 & -5 & 1 & & \\
\hline (31) & -3 & -2 & 9 & 1 & -6 & -1 & & \\
\hline (32) & -3 & -2 & 10 & -1 & -7 & 1 & & \\
\hline (33) & -2 & -5 & 7 & 8 & -3 & -1 & & \\
\hline (34) & -2 & -5 & 8 & 7 & -7 & -1 & & \\
\hline (35) & -2 & -5 & 8 & 8 & -7 & -4 & & \\
\hline (36) & -2 & -5 & 9 & 6 & -9 & 1 & & \\
\hline (37) & -2 & -4 & 7 & 4 & -4 & -1 & & \\
\hline (38) & -2 & -4 & 8 & 2 & -5 & 1 & & \\
\hline (39) & -1 & -6 & 4 & 9 & -3 & -1 & & \\
\hline (40) & -1 & -6 & 5 & 9 & -6 & -1 & & \\
\hline (41) & -1 & -5 & 4 & 5 & -2 & -1 & & \\
\hline (42) & -3 & -3 & 11 & 3 & -10 & -1 & 1 & \\
\hline (43) & -3 & -3 & 11 & 3 & -9 & -2 & 1 & \\
\hline (44) & -3 & -3 & 12 & 2 & -13 & 0 & 3 & \\
\hline (45) & -3 & -3 & 13 & 0 & -14 & 2 & 3 & \\
\hline (46) & -2 & -6 & 11 & 11 & -17 & -6 & 7 & \\
\hline (47) & -2 & -5 & 8 & 8 & -7 & -3 & 1 & \\
\hline (48) & -2 & -5 & 9 & 7 & -10 & -2 & 1 & \\
\hline (49) & -2 & -5 & 9 & 7 & -9 & -3 & 1 & \\
\hline (50) & -1 & -7 & 4 & 15 & -3 & -9 & -1 & \\
\hline (51) & -1 & -7 & 4 & 15 & -2 & -8 & -1 & \\
\hline (52) & -1 & -7 & 5 & 15 & -6 & -9 & 1 & \\
\hline (53) & -1 & -7 & 5 & 15 & -5 & -10 & -1 & \\
\hline (54) & -1 & -7 & 6 & 13 & -9 & -3 & 1 & \\
\hline (55) & -1 & -6 & 4 & 10 & -4 & -4 & 1 & \\
\hline (56) & 0 & -8 & 0 & 19 & 0 & -12 & -1 & \\
\hline (57) & -4 & -2 & 21 & -6 & -33 & 12 & 13 & -1 \\
\hline (58) & -4 & -1 & 17 & -6 & -21 & 8 & 6 & -1 \\
\hline (59) & -4 & -1 & 17 & -5 & -23 & 6 & 9 & -1 \\
\hline (60) & -4 & -1 & 18 & -9 & -21 & 13 & 3 & -1 \\
\hline (61) & -3 & -5 & 18 & 7 & -33 & -3 & 18 & 1 \\
\hline (62) & -3 & -4 & 14 & 6 & -19 & -5 & 6 & 1 \\
\hline (63) & -3 & -4 & 15 & 4 & -22 & 0 & 8 & -1 \\
\hline (64) & -3 & -4 & 15 & 4 & -22 & 0 & 9 & -1 \\
\hline (65) & -3 & -4 & 15 & 4 & -21 & -2 & 8 & 1 \\
\hline (66) & -2 & -7 & 11 & 18 & -16 & -18 & 3 & 3 \\
\hline (67) & -2 & -7 & 11 & 18 & -15 & -18 & 0 & 1 \\
\hline (68) & -2 & -7 & 12 & 17 & -21 & -16 & 10 & 5 \\
\hline (69) & -2 & -7 & 12 & 17 & -21 & -15 & 9 & 1 \\
\hline (70) & -2 & -6 & 10 & 12 & -13 & -8 & 3 & 1 \\
\hline (71) & -2 & -6 & 11 & 11 & -17 & -6 & 6 & 1 \\
\hline (72) & -2 & -6 & 11 & 11 & -16 & -7 & 5 & 1 \\
\hline (73) & -1 & -8 & 5 & 21 & -6 & -18 & 2 & 3 \\
\hline (74) & -1 & -7 & 5 & 15 & -7 & -10 & 2 & 1 \\
\hline (75) & -1 & -7 & 5 & 15 & -6 & -10 & 1 & 1 \\
\hline (76) & -1 & -7 & 4 & 15 & -3 & -9 & 0 & 1 \\
\hline (77) & 0 & -8 & -1 & 20 & 4 & -16 & -3 & 2 \\
\hline
\end{tabular}

\section{Appendix A: The set $\mathcal{N}$}

The list we present here follows the lexicographic ordering $\prec$. The leading coefficient, 1 , is omitted; for example, the first line corresponds to $x^{2}-x-3$. The choice of representatives maintains Robinson's criterion: the average of roots has to lie in $\left[0, \frac{1}{2}\right]$ (see [8] for further details). 


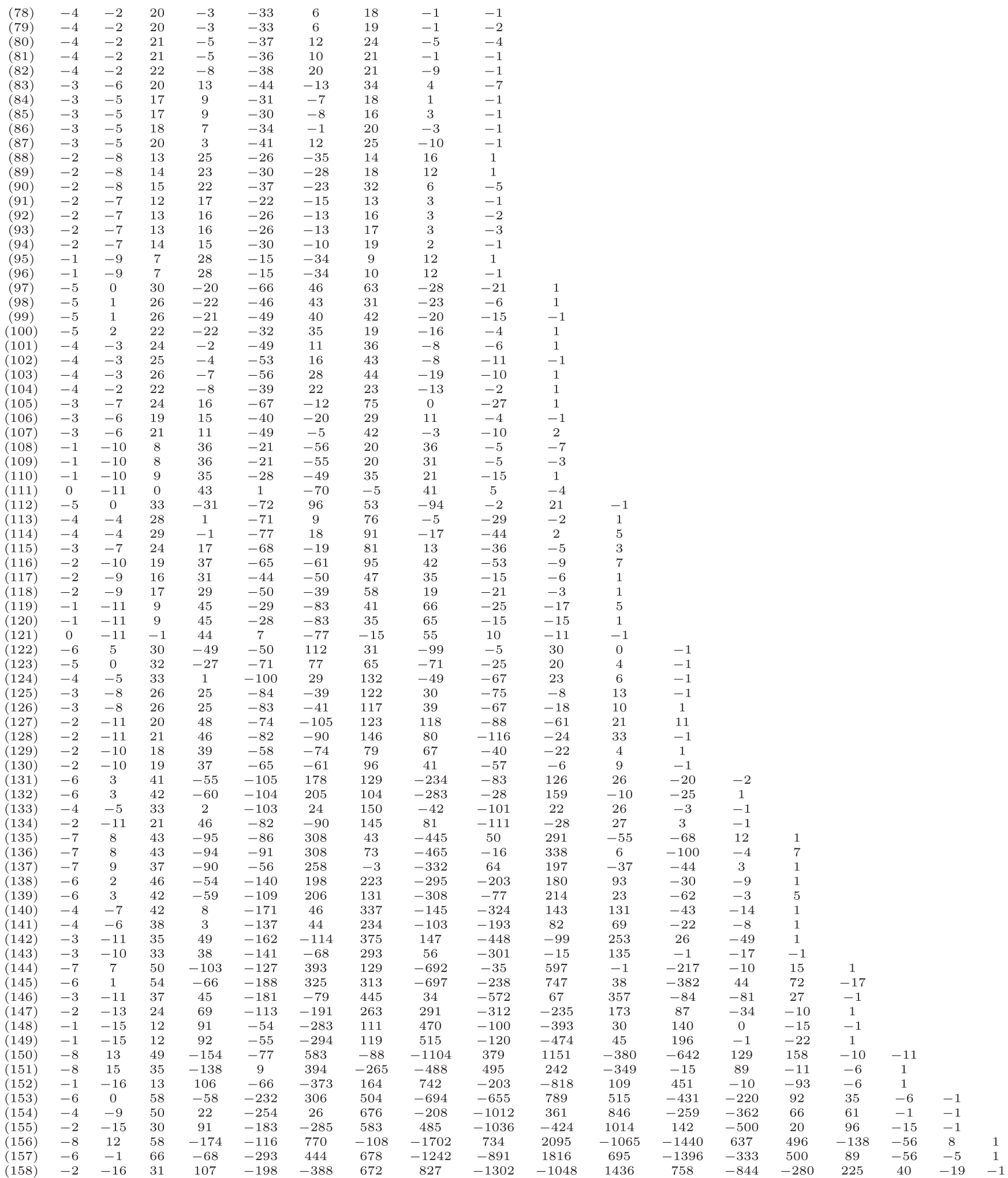




\section{Appendix B: Data for the proof of Proposition 2}

According to the first line of the list in this Appendix, $f_{15}$ is a factor of $f_{7} f_{8}-f_{2}+f_{1}-1$, and similarly with the next lines. In the two lines having a single asterisk we actually found $f_{i}(-x)$; curiously enough, we did not find other representatives of the form $f_{i}(-x)$, nor other forms like $f_{i}(1-x), f_{i}(x-1)$, etc. The double asterisk witnesses the presence of some Kronecker polynomials. There are 6 such polynomials-we used Greek letters as symbolic indices:

$$
\begin{aligned}
f_{\alpha}= & x^{6}-x^{5}-5 x^{4}+4 x^{3}+6 x^{2}-3 x-1 . \\
f_{\beta}= & x^{3}-3 x+1 \\
f_{\gamma}= & x^{4}-4 x^{2}+1 \\
f_{\delta}= & x \\
f_{\varepsilon}= & x^{2}-x-1 . \\
f_{\zeta}= & x^{11}-x^{10}-10 x^{9}+9 x^{8}+36 x^{7}-28 x^{6}-56 x^{5}+35 x^{4}+35 x^{3} \\
& -15 x^{2}-6 x+1 .
\end{aligned}
$$

Here follows the list.

\begin{tabular}{cccccc}
\hline Degree & 5 & & & \\
7 & 8 & 2 & 1 & $\rightarrow$ & 15 \\
8 & 3 & 1 & 10 & $\rightarrow$ & 16 \\
11 & 8 & 2 & 4 & $\rightarrow$ & 17 \\
6 & 12 & 10 & 12 & $\rightarrow$ & 18 \\
11 & 2 & 2 & 10 & $\rightarrow$ & 19 \\
11 & 12 & 8 & 12 & $\rightarrow$ & 20 \\
8 & 2 & 12 & 8 & $\rightarrow$ & 21 \\
14 & 8 & 12 & 8 & $\rightarrow$ & 22 \\
11 & 2 & 7 & 12 & $\rightarrow$ & 23 \\
14 & 11 & 1 & 14 & $\rightarrow$ & 24 \\
11 & 3 & 14 & 5 & $\rightarrow$ & $25^{*}$ \\
7 & 3 & 2 & 5 & $\rightarrow$ & 26 \\
8 & 3 & 11 & 5 & $\rightarrow$ & 27 \\
13 & 2 & 12 & 8 & $\rightarrow$ & 28
\end{tabular}

Degree 6

$\begin{array}{cccccc}8 & 1 & 3 & 2 & \rightarrow & 29 \\ 6 & 5 & 5 & 1 & \rightarrow & 30 \\ 12 & 8 & 14 & 10 & \rightarrow & 31 \\ 8 & 1 & 4 & 13 & \rightarrow & 32 \\ 12 & 1 & 3 & 2 & \rightarrow & 33 \\ 12 & 2 & 14 & 9 & \rightarrow & 34 \\ 23 & 7 & 11 & 1 & \rightarrow & 35 \\ 14 & 2 & 7 & 10 & \rightarrow & 36 \\ 11 & 8 & 8 & 12 & \rightarrow & 37 \\ 23 & 13 & 7 & 12 & \rightarrow & 38 \\ 22 & 8 & 16 & 8 & \rightarrow & 39 \\ 22 & 23 & 12 & 1 & \rightarrow & 40 \\ 13 & 8 & 12 & 2 & \rightarrow & 41\end{array}$

Degree 7

$\begin{array}{llllll}30 & 8 & 37 & 30 & \rightarrow & 42\end{array}$

$\begin{array}{llllll}23 & 6 & 19 & 22 & \rightarrow & 43\end{array}$

$\begin{array}{llllll}8 & 2 & 14 & 13 & \rightarrow & 44\end{array}$

$\begin{array}{llllll}11 & 12 & 12 & 10 & \rightarrow & 45\end{array}$

$\begin{array}{llllll}14 & 11 & 1 & 10 & \rightarrow & 46\end{array}$

$\begin{array}{llllll}7 & 8 & 2 & 4 & \rightarrow & 47\end{array}$

$\begin{array}{llllll}17 & 12 & 1 & 8 & \rightarrow & 48\end{array}$

$\begin{array}{llllll}20 & 23 & 1 & 4 & \rightarrow & 49\end{array}$

$\begin{array}{llllll}23 & 8 & 23 & 7 & \rightarrow & 50\end{array}$

$\begin{array}{llllll}12 & 4 & 1 & 3 & \rightarrow & 51\end{array}$

$\begin{array}{llllll}114 & 3 & 83 & 2 & \rightarrow & 126\end{array}$

$\begin{array}{llllll}72 & 27 & 37 & 33 & \rightarrow & 127\end{array}$

$\begin{array}{llllll}96 & 65 & 93 & 37 & \rightarrow & 128\end{array}$

$\begin{array}{llllll}115 & 51 & 21 & 18 & \rightarrow & 129\end{array}$

$\begin{array}{llllll}116 & 55 & 48 & 91 & \rightarrow & 130\end{array}$

Degree 13

$\begin{array}{cccccc}63 & 59 & 11 & 8 & \rightarrow & 131 \\ 62 & 47 & 48 & 55 & \rightarrow & 132 \\ 102 & 48 & 10 & 45 & \rightarrow & 133 \\ 117 & 75 & 10 & 11 & \rightarrow & 134\end{array}$

Degree 14

$\begin{array}{llllll}122 & 5 & 1 & 97 & \rightarrow & 135\end{array}$

$\begin{array}{llllll}122 & 98 & 4 & 2 & \rightarrow & 136\end{array}$

$\begin{array}{cccccc}24 & 23 & 2 & 14 & \rightarrow & 52 \\ 24 & 23 & 11 & 26 & \rightarrow & 53 \\ 26 & 20 & 23 & 27 & \rightarrow & 54 \\ 37 & 24 & 12 & 20 & \rightarrow & 55 \\ 40 & 13 & 33 & 5 & \rightarrow & 56\end{array}$

Degree 8

$\begin{array}{llllll}31 & 19 & 2 & 32 & \rightarrow & 57\end{array}$

$\begin{array}{llllll}29 & 30 & 34 & 21 & \rightarrow & 58\end{array}$

$\begin{array}{llllll}31 & 8 & 3 & 2 & \rightarrow & 59\end{array}$

$\begin{array}{llllll}31 & 7 & 1 & 7 & \rightarrow & 60\end{array}$

$\begin{array}{llllll}27 & 5 & 10 & 53 & \rightarrow & 61\end{array}$

$\begin{array}{llllll}10 & 8 & 7 & 6 & \rightarrow & 62\end{array}$

$\begin{array}{llllll}11 & 8 & 14 & 3 & \rightarrow & 63\end{array}$

$\begin{array}{llllll}11 & 8 & 13 & 2 & \rightarrow & 64\end{array}$

$\begin{array}{llllll}29 & 37 & 31 & 12 & \rightarrow & 65\end{array}$

$\begin{array}{llllll}7 & 14 & 22 & 6 & \rightarrow & 66\end{array}$

$\begin{array}{llllll}17 & 12 & 35 & 30 & \rightarrow & 67\end{array}$

$\begin{array}{cccccc}37 & 7 & 8 & 11 & \rightarrow & 68\end{array}$

$\begin{array}{llllll}37 & 17 & 32 & 31 & \rightarrow & 69\end{array}$

$\begin{array}{llllll}37 & 17 & 23 & 20 & \rightarrow & 70\end{array}$

$\begin{array}{llllll}37 & 11 & 31 & 30 & \rightarrow & 71\end{array}$

$\begin{array}{llllll}39 & 35 & 37 & 6 & \rightarrow & 72\end{array}$

$\begin{array}{llllll}13 & 12 & 7 & 6 & \rightarrow & 73\end{array}$

$\begin{array}{llllll}38 & 2 & 1 & 17 & \rightarrow & 74\end{array}$

$\begin{array}{llllll}39 & 41 & 22 & 28 & \rightarrow & 75\end{array}$

$\begin{array}{llllll}37 & 2 & 32 & 30 & \rightarrow & 76\end{array}$

$\begin{array}{llllll}23 & 27 & 12 & 28 & \rightarrow & 77\end{array}$

Degree 9

$\begin{array}{llllll}49 & 5 & 50 & 15 & \rightarrow & 78\end{array}$

$\begin{array}{llllll}58 & 18 & 12 & 8 & \rightarrow & 79\end{array}$

$\begin{array}{llllll}62 & 8 & 22 & 15 & \rightarrow & 80\end{array}$

$\begin{array}{llllll}30 & 12 & 31 & 33 & \rightarrow & 81\end{array}$

$\begin{array}{llllll}39 & 32 & 37 & 30 & \rightarrow & 82\end{array}$

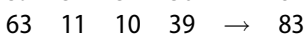

$\begin{array}{llllll}63 & 42 & 64 & 72 & \rightarrow & 84\end{array}$

$\begin{array}{llllll}63 & 59 & 11 & 61 & \rightarrow & 85\end{array}$

$\begin{array}{llllll}71 & 2 & 40 & 47 & \rightarrow & 86\end{array}$

$\begin{array}{llllll}71 & 38 & 48 & 38 & \rightarrow & 87\end{array}$

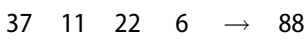

$\begin{array}{llllll}49 & 23 & 3 & 2 & \rightarrow & 89\end{array}$

$\begin{array}{llllll}122 & 97 & 59 & 8 & \rightarrow & 137\end{array}$

$\begin{array}{llllll}131 & 31 & 44 & 31 & \rightarrow & 138\end{array}$

$\begin{array}{llllll}85 & 59 & 16 & 19 & \rightarrow & 139\end{array}$

$\begin{array}{llllll}103 & 73 & 2 & 48 & \rightarrow & 140\end{array}$

$\begin{array}{llllll}107 & 73 & 2 & 39 & \rightarrow & 141\end{array}$

$117 \quad 40 \quad 40 \quad \beta \quad \rightarrow \quad 143^{(* *)}$

Degree 15

$\begin{array}{cccccc}81 & 29 & 1 & 15 & \rightarrow & 144 \\ 104 & 88 & 64 & 96 & \rightarrow & 145 \\ 119 & 96 & 14 & 13 & \rightarrow & 146 \\ 143 & 37 & 72 & 60 & \rightarrow & 147 \\ 120 & 14 & \gamma & \delta & \rightarrow & 148^{(* *)} \\ 77 & 52 & 7 & 6 & \rightarrow & 149\end{array}$

$\begin{array}{cccccc}49 & 23 & 4 & 2 & \rightarrow & 90 \\ 37 & 7 & 20 & 11 & \rightarrow & 91 \\ 37 & 23 & 24 & 8 & \rightarrow & 92 \\ 37 & 23 & 17 & 8 & \rightarrow & 93 \\ 75 & 7 & 37 & 34 & \rightarrow & 94 \\ 49 & 27 & 9 & 55 & \rightarrow & 95 \\ 16 & 24 & 7 & 54 & \rightarrow & 96\end{array}$

$\begin{array}{llllll}16 & 24 & 7 & 54 & \rightarrow & 96\end{array}$

Degree 10

$\begin{array}{llllll}30 & 5 & 2 & 3 & \rightarrow & 97\end{array}$

$\begin{array}{llllll}29 & 31 & 30 & 29 & \rightarrow & 98\end{array}$

$\begin{array}{llllll}58 & 31 & 21 & 18 & \rightarrow & 99\end{array}$

$\begin{array}{llllll}62 & 16 & 67 & 62 & \rightarrow & 100\end{array}$

$\begin{array}{llllll}57 & 33 & 63 & 44 & \rightarrow & 101\end{array}$

$\begin{array}{llllll}68 & 70 & 71 & 19 & \rightarrow & 102\end{array}$

$\begin{array}{llllll}19 & 17 & 7 & 15 & \rightarrow & 103\end{array}$

$\begin{array}{llllll}80 & 2 & 10 & 13 & \rightarrow & 104\end{array}$

$\begin{array}{llllll}88 & 11 & 48 & 1 & \rightarrow & 105\end{array}$

$\begin{array}{llllll}88 & 30 & 6 & 5 & \rightarrow & 106\end{array}$

$\begin{array}{llllll}19 & 23 & 1 & 4 & \rightarrow & 107\end{array}$

$\begin{array}{llllll}96 & 49 & 1 & 96 & \rightarrow & 108\end{array}$

$\begin{array}{llllll}96 & 75 & 17 & 24 & \rightarrow & 109\end{array}$

$\begin{array}{llllll}94 & 26 & 24 & 56 & \rightarrow & 110\end{array}$

$94 \quad 26 \quad 40 \quad 56 \rightarrow 111^{(*)}$

Degree 11

$\begin{array}{llllll}102 & 63 & 6 & 7 & \rightarrow & 112\end{array}$

$\begin{array}{llllll}102 & 63 & 71 & 11 & \rightarrow & 113\end{array}$

$\begin{array}{llllll}46 & 45 & 48 & 35 & \rightarrow & 114\end{array}$

$\begin{array}{llllll}43 & 46 & 32 & 2 & \rightarrow & 115\end{array}$

$\begin{array}{llllll}77 & 11 & 11 & 5 & \rightarrow & 116\end{array}$

$\begin{array}{llllll}91 & 37 & 44 & 11 & \rightarrow & 117\end{array}$

$\begin{array}{llllll}107 & 4 & 71 & 89 & \rightarrow & 118\end{array}$

$\begin{array}{llllll}108 & 2 & 14 & 96 & \rightarrow & 119\end{array}$

$40 \quad \propto \quad 39 \quad 15 \rightarrow 120^{(* *)}$

$\begin{array}{llllll}94 & 27 & 64 & 29 & \rightarrow & 121\end{array}$

Degree 12

$\begin{array}{cccccc}59 & 29 & 3 & 2 & \rightarrow & 122 \\ 70 & 64 & 72 & 65 & \rightarrow & 123 \\ 69 & 61 & 48 & 17 & \rightarrow & 124 \\ 112 & 47 & 63 & 57 & \rightarrow & 125\end{array}$

Degree 16

$\begin{array}{llllll}100 & 58 & 4 & 2 & \rightarrow & 150\end{array}$

$\begin{array}{llllll}100 & 58 & \varepsilon & 122 & \rightarrow & 151^{(* *)}\end{array}$

Degree 17

$\begin{array}{llllll}138 & 104 & 31 & \varepsilon & \rightarrow & 153^{(* *)}\end{array}$

$\begin{array}{llllll}\zeta & 96 & 22 & 27 & \rightarrow & 155^{(* *)}\end{array}$

Degree 18

$\begin{array}{lllllll}148 & 109 & 28 & \varepsilon & \rightarrow & 158^{(* *)}\end{array}$ 


\section{Acknowledgements}

We are grateful to the anonymous referee for valuable comments and suggestions. This article was prepared with the financial support of Sapienza University of Rome, Progetti di Ateneo.

\section{References}

[1] Capparelli, S., Del Fra, A, Sciò, C. (2009). On the span of polynomials with integer coefficients. Math. Comp. 79:967-981. DOI: 10.1090/S0025-5718-09-02292-3

[2] El Otmani, S., Maul, A., Rhin, G, Sac-Épée, J. M. (2013). Integer linear programming applied to determining monic hyperbolic irreducible polynomials with integer coefficients and span less than 4 . J. Théor. Nombres Bordeaux. 25:71-78. DOI: 10.5802/jtnb.826

[3] Flammang, V., Rhin, G, Wu, Q. (2011). The totally real algebraic integers with diameter less than 4. Mosc. J. Comb. Number Theory. 1:17-25.

[4] Kronecker, L. (1857). Zwei Sätze über Gleichungen mit ganzzahligen Coefficienten. J. Reine Angew. Math. 53:173-175.

[5] Marden, M. (1949). Geometry of Polynomials. Providence, RI: AMS. Reprinted 1989.

[6] McKee, J. (2010). Small-span characteristic polynomials of integer symmetric matrices. In: Hanrot, G., Morain, F., Thomé, E., eds. Algorithmic Number Theory, Lecture Notes in Computer Science, vol. 6197. Berlin: Springer, p. 270-284.
[7] Robinson, R. (1962). Intervals containing infinitely many sets of conjugate algebraic integers. In: Gabor Szegö et al., eds. Studies in Mathematical Analysis and Related Topics: Essays in Honor of George Pólya. Stanford, CA: Stanford University Press, p. 305-315.

[8] Robinson, R. (1964). Algebraic equations with Span less than 4. Math. Comput. 18:547-559. DOI: 10.2307/2002941

[9] Robinson, R. (1964). Intervals containing infinitely many sets of conjugate algebraic units. Ann. Math. 80:411-428. DOI: 10. 2307/1970656

[10] Salem, R. (1944). A remarkable class of algebraic integers. Proof of a conjecture of Vijayaraghavan. Duke Math. J. 11:103-108. DOI: $10.1215 /$ S0012-7094-44-01111-7

[11] Salem, R. (1945). Power series with integral coefficients. Duke Math. J. 12:153-172. DOI: 10.1215/S0012-7094-4501213-0

[12] Salem, R. (1963). Algebraic Numbers and Fourier Analysis. Boston, MA: D.C. Heath and Co.

[13] Schur, I. (1918). Über die Verteilung der Wurzeln bei gewissen algebraischen Gleichungen mit ganzzahligen Koeffizienten. Math. Z. 1:377-402. DOI: 10.1007/BF01465096

[14] Smyth, C. (2015). Seventy years of Salem numbers: a survey. Bull. Lond. Math. Soc. 47(3):379-395. DOI: 10.1112/blms/ bdv027

[15] Walsh, J. L. (1922). On the location of the roots of certain types of polynomials. Trans. Am. Math. Soc. 24:163-180. DOI: 10 . 1090/S0002-9947-1922-1501220-0 